\title{
Periconceptional and antenatal nutritional supplement use in Irish women: data from the improved study
}

\author{
L. Kelliher ${ }^{1,2}$, Á. Hennessy ${ }^{1,2}$, F. McCarthy ${ }^{2,3}$ and M.E. Kiely ${ }^{1,2}$ \\ ${ }^{1}$ Cork Centre for Vitamin D and Nutrition Research, School of Food and Nutritional Sciences, University College \\ Cork, Cork, Ireland, \\ ${ }^{2}$ The Irish Centre for Maternal and Child Health Research (INFANT), University College Cork, Cork, Ireland and \\ ${ }^{3}$ Department of Obstetrics and Gynaecology, University College Cork, Cork, Ireland
}

Suboptimal micronutrient status in the early antenatal period can increase the risk of adverse perinatal outcomes, specifically neural tube defects and suboptimal neurological development ${ }^{(1)}$. The aim of this study was to describe the prevalence of nutritional supplement use in the periconceptional and antenatal periods in Irish women participating in the IMproved Pregnancy Outcomes by Early Detection (IMPROvED) Study (http://www.clinicaltrials.gov: ID NCT01891240).

Participants $(n=1509)$ were healthy, nulliparous women with a low-risk, singleton pregnancy, receiving antenatal care at Cork University Maternity Hospital. Women provided written informed consent during their first trimester and completed a series of clinical- and questionnaire-based assessments at 11-, 15-, 20- and 33-weeks' gestation ( $\mathrm{n}=409,1488$, 1445 and 680, respectively). Brand-level data on the use of nutritional supplements were reported pre-conception and during the first trimester. Data were analysed using IBM SPSS (Version 27, IBM Corp, Armonk NY). Demographic and health and lifestyle characteristics were compared between users and non-users of nutritional supplements using Pearson's Chi-Square. An alpha of $<0.05$ was considered statistically significant.

The median (IQR) maternal age was 31 (5) years, 70\% of women completed third-level education, $89 \%$ were in full- or part-time employment and $93 \%$ were married or in a relationship. Smoking was reported by $14 \%$ of women at their first study visit, while $3 \%$ reported consuming alcohol during pregnancy. The median (IQR) maternal BMI at 15 weeks' gestation was $24.5(4.7) \mathrm{kg} / \mathrm{m}^{2}$.

Prior to conception, the prevalence of folic acid supplementation was $65 \%$. Among users, the median (IQR) duration of folic acid supplement use was 4 (7) months, and 75\% of women used folic acid for at least 3 months before conception as recommended by the Irish Health Service Executive ${ }^{(2)}$. Folic acid supplementation increased to $99 \%$ in the first trimester and $98 \%$ met the RDA of $400 \mu \mathrm{g} / \mathrm{d}$. By 15 weeks' gestation, the prevalence of folic acid supplementation decreased to $87 \%$.

The prevalence of multivitamin supplement use in the periconceptional period was low (30\%) and $42 \%$ of users reported using a supplement designed to support fertility and conception. Multivitamin supplement use increased to $58 \%$ in the first trimester of pregnancy and to $70 \%$ by 15 weeks' gestation. Overall, $70 \%$ and $67 \%$ of women used vitamin D- and iodine-containing supplements, respectively during pregnancy. Median intakes of supplemental vitamin D and iodine were $10 \mu \mathrm{g} / \mathrm{d}$ and $150 \mu \mathrm{g} / \mathrm{d}$, respectively. Women who were older, non-smokers and had completed third-level education were significantly more likely to use folic acid and multivitamin supplements prior to conception and during their pregnancy.

Our findings show periconceptional and antenatal supplement use has remained similar to prevalence rates reported in the SCOPE Ireland Pregnancy Study ${ }^{(3)}$ and is linked with socio-demographic background and the intention to conceive.

\section{Acknowledgments}

This work was supported by a Science Foundation Ireland Starting Investigator award to ÁH [Functional indicators of iodine status in pregnancy - an outcome-driven, personalised nutrition approach (18/SIRG/5575)]

\section{References}

1. Georgieff MK, Ramel SE \& Cusick SE (2018) Acta Paediatr 107, 1310-21.

2. Health Service Executive (2019) Clinical Practice Guidance: Nutrition During Pregnancy, HSE.

3. O’ Callaghan K, Hennessy Á, Kenny LC et al. (2015) Proc Nutr Soc 74, E259. 